\title{
Targeting CD205 with the antibody drug conjugate MEN1309/0BT076 is an active new therapeutic strategy in lymphoma models
}

Haematologica 2020

Volume 105(11):2584-2591

\section{Correspondence:}

FRANCESCO BERTONI

francesco.bertoni@ior.usi.ch

Received: May 16, 2019.

Accepted: January 2, 2020.

Pre-published: January 9, 2020.

doi:10.3324/haematol.2019.227215

(C)2020 Ferrata Storti Foundation

Material published in Haematologica is covered by copyright. All rights are reserved to the Ferrata Storti Foundation. Use of published material is allowed under the following terms and conditions:

https://creativecommons.org/licenses/by-nc/4.0/legalcode. Copies of published material are allowed for personal or internal use. Sharing published material for non-commercial purposes is subject to the following conditions:

https://creativecommons.org/licenses/by-nc/4.0/legalcode, sect. 3. Reproducing and sharing published material for commercial purposes is not allowed without permission in writing from the publisher.

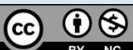

\author{
Eugenio Gaudio, ${ }^{1 *}$ Chiara Tarantelli ${ }^{1 *}$ Filippo Spriano, ${ }^{1}$ Francesca Guidetti, ${ }^{1}$ \\ Giulio Sartori, ${ }^{1}$ Roberta Bordone,${ }^{2}$ Alberto J. Arribas,${ }^{1}$ Luciano Cascione,,${ }^{1,3}$ \\ Mario Bigioni, ${ }^{4}$ Giuseppe Merlino,${ }^{4}$ Alessio Fiascarelli, ${ }^{4}$ Alessandro Bressan,${ }^{4}$ \\ Afua Adjeiwaa Mensah, ${ }^{1}$ Gaetanina Golino, ${ }^{1}$ Renzo Lucchini, ${ }^{5}$ \\ Elena Bernasconi, ${ }^{1}$ Davide Rossi, ${ }^{1,2}$ Emanuele Zucca, ${ }^{2}$ Georg Stussi, ${ }^{2}$ \\ Anastasios Stathis, ${ }^{2}$ Robert S. Boyd,${ }^{6}$ Rachel L. Dusek, ${ }^{6}$ Arnima Bisht, ${ }^{7}$ \\ Nickolas Attanasio, ${ }^{7}$ Christian Rohlff, ${ }^{7}$ Andrea Pellacani, ${ }^{8}$ Monica Binaschi ${ }^{4}$ \\ and Francesco Bertoni ${ }^{1,2}$
}

\begin{abstract}
${ }^{1}$ Università della Svizzera italiana, Istituto Oncologico di Ricerca, Bellinzona, Switzerland ${ }^{2}$ Oncology Institute of Southern Switzerland, Bellinzona, Switzerland; ${ }^{3}$ Swiss Institute of Bioinformatics (SIB), Lausanne, Switzerland; ${ }^{4}$ Menarini Ricerche S.p.A., Pomezia, Italy; ${ }^{5}$ Laboratorio di Diagnostica Molecolare, Dipartimento di Medicina di Laboratorio EOLAB, Bellinzona, Switzerland; ${ }^{6}$ Oxford BioTherapeutics Ltd., Abingdon, UK; ${ }^{7}$ Oxford BioTherapeutics Inc., San Jose, CA, USA and ${ }^{8}$ Menarini Ricerche S.p.A - Menarini Group, Florence, Italy
\end{abstract}

${ }^{\star} E G$ and $C T$ contributed equally as co-first authors.

\section{ABSTRACT}

A ntibody drug conjugates represent an important class of anti-cancer drugs in both solid tumors and hematologic cancers. Here, we report preclinical data on the anti-tumor activity of the first-in-class antibody drug conjugate MEN1309/OBT076 targeting CD205. The study included preclinical in vitro activity screening on a large panel of cell lines, both as single agent and in combination, and validation experiments on in vivo models. CD205 was first shown frequently expressed in lymphomas, leukemias and multiple myeloma by immunohistochemistry on tissue microarrays. Anti-tumor activity of MEN1309/OBT076 as single agent was then shown across 42 B-cell lymphoma cell lines with a median IC 50 of 200 $\mathrm{pM}$ and induction of apoptosis in 25 of $42(59.5 \%)$ of the cases. The activity appeared highly correlated with its target expression. After in vivo validation as the single agent, the antibody drug conjugate synergized with the BCL2 inhibitor venetoclax and the anti-CD20 monoclonal antibody rituximab. The first-in-class antibody drug targeting CD205, MEN1309/OBT076, demonstrated strong pre-clinical anti-tumor activity in lymphoma, warranting further investigations as a single agent and in combination.

\section{Introduction}

Antibody drug conjugates $(\mathrm{ADC})$ represent a class of anti-cancer agents that can have an important impact on the clinical outcome of cancer patients, as exemplified by brentuximab vedotin for Hodgkin lymphoma patients or ado-trastuzumabemtansine (T-DM1) for breast cancer patients. ${ }^{1}$ In lymphomas, different surface proteins (CD19, CD37, CD79B, CD25) have provided targets for active ADC. ${ }^{1} \mathrm{CD} 205$, encoded by the LY75 gene (Lymphocyte antigen 75, DEC-205), is a surface multilectin receptor with a cytoplasmatic domain containing protein motifs crucial for endocytosis and internalization upon ligation. Although its biologic role has not been fully defined, it is known to act as a surface receptor for apoptotic and necrotic cells, ${ }^{2,3}$ leading to antigen uptake and processing. CD205 is expressed in hematopoietic cells, mainly by antigen presenting cells (APC), but also in other tissues, including solid tumors. ${ }^{48} \mathrm{CD} 205$ presents a rapid internalization rate and a favorable profile in terms of differential expression between neoplastic and healthy tissues, and is a potential new target for ADC. ${ }^{7}$ MEN1309/OBT076 is a novel humanized IgG1 anti- 
body directed against CD205, with no direct anti-tumor effect, conjugated through a cleavable $\mathrm{N}$-succinimidyl-4-(2pyridyldithio) butanoate linker to the potent maytansinoid microtubule disruptor DM4. ${ }^{7}$ The ADC has shown potent in vivo anti-tumor activity with durable responses and complete tumor regressions in many models derived from triple negative breast cancer, pancreatic and bladder cancer cell lines and primary cells. 'MEN1309/OBT076 is now under early clinical investigation for patients with solid tumors and lymphoma (CD205-Shuttle study; clinicaltrials.gov identifier: NCT03403725). ${ }^{9}$ Here, we present the first preclinical data sustaining CD205 as a novel therapeutic target for lymphomas.

\section{Methods}

\section{Cell lines}

A total of 42 lymphoma cell lines derived from germinal center B-cell type (GCB, $n=17)$ or activated B-cell-like $(n=7)$ diffuse large B-cell lymphoma (DLBCL), mantle cell lymphoma (MCL, $n=10)$, marginal zone lymphoma (MZL, $\mathrm{n}=6$ ), and chronic lymphocytic leukemia (CLL, $n=2$ ) were used and cultured as previously described. ${ }^{10}$ Cell line identity was validated by STR DNA fingerprinting using the Promega GenePrint 10 System kit (B9510) (Online Supplementary Table S1). BCL2, MYC and TP53 status were defined as previously described. ${ }^{11}$

\section{Compounds}

MEN1309/OBT076, MBH1309 and IgG-DM4 for proliferation assay and MEN1309-PE for FACS analysis were provided by Menarini. Idelalisib, bortezomib, lenalidomide, venetoclax were purchased from Selleckchem (Houston, TX, USA) and rituximab from Roche (Basel, Switzerland).

\section{Proliferation and apoptosis assays}

Anti-proliferative activity of MEN1309/OBT076 or IgG-DM4 was assessed as before ${ }^{12}$ and are described in the Online Supplementary Appendix. Methods for apoptosis detection are defined in the Online Supplementary Appendix. Peripheral blood mononuclear cells (PBMC) from healthy donors were isolated by using the Ficoll-Paque PLUS (Ge Heathcare Lifesciences) reagent according to its guidelines. $\mathrm{CD} 19^{+} \mathrm{B}$-cell lymphocytes were isolated from PBMC using the CD19 MicroBeads (MACS Miltenyi Biotec). In vitro combinations were assessed as previously described. ${ }^{10,12}$ Based on the Chou-Talalay Combination Index, ${ }^{13}$ the effect of the combinations was defined as beneficial if synergistic $(<0.9)$ or additive (0-9-1.1).

\section{Western blotting analysis}

Protein extraction, separation and immunoblotting were performed as previously described. ${ }^{10}$ The following antibodies were used: anti- $\beta$-Tubulin (cst-2146, Cell Signaling Technology), antiPARP1 (sc-8007, Santa Cruz Biotechnology), anti-BCL2 (sc-492), anti-MCL1 (cst-5453), anti- $\beta$ BCLXL (sc-8392), and anti-CD20 (ab9475, Abcam).

\section{Immunohistochemistry \\ Immunohistochemical staining was performed as described in the Online Supplementary Appendix.}

\section{CD205 surface expression by cytofluorimetry}

CD205 expression was determined by flow cytofluorimetry (FACS) on fresh cells, as described in the Online Supplementary Appendix.

\section{Real-time-polymerase chain reaction}

Total RNA was extracted from cells by using TRIZOL. cDNA was prepared by using the Super script double strand cDNA synthesis kit (Thermo Fisher Scientific, Waltham, MA, USA). The expression levels of both CD205 and the reported intergenically spliced forms were analyzed as described in the Online Supplementary Appendix. RNA expression levels obtained with the HumanHT-12 v4 Expression BeadChip (Illumina, San Diego, CA, USA) were retrieved from our previous publication (GSE94669). ${ }^{10}$

\section{Data mining}

Associations in two-way tables were tested for statistical significance using either the $\chi^{2}$ test or Fisher exact test (two-tailed), as appropriate. Binomial exact $95 \%$ confidence intervals $(95 \% \mathrm{CI})$ were calculated for median percentages. Differences in $\mathrm{IC}_{50}$ values among subtypes were calculated using the Wilcoxon rank-sum test. Baseline gene expression levels of CD205 and of CD302 were extracted from the GSE9466910 dataset obtained using the HumanHT-12 v4 Expression BeadChip (Illumina, San Diego, CA, USA). The degree of correlation among genes was calculated by standard Pearson correlation coefficients. $P<0.05$ was considered statistically significant. Statistical analyses were conducted using Stata/SE 12.1 for Mac (Stata Corporation, College Station, TX, USA).

\section{Animal studies}

Mice maintenance and animal experiments were performed under the institutional guidelines established for the Animal Facility and with study protocols approved by the local Cantonal Veterinary Authority (license TI-22-2015). Methods are described in the Online Supplementary Appendix.

\section{Results}

Cluster of differentiation 205 (CD205) is expressed in most diffuse large B-cell lymphoma

Expression of CD205 was assessed using immunohistochemistry (IHC) on formalin-fixed paraffin embedded sections of clinical specimens of hematologic cancers derived from lymphomas ( $\mathrm{n}=370)$, acute myeloid leukemia $(\mathrm{n}=26)$, and multiple myeloma $(n=14)$ (Table 1). The antigen CD205 was expressed in the vast majority of the cases, and in entities comprising larger number of samples (DLBCL, MALT lymphomas) expression was detected in $73 \%$ and $88 \%$ of the cases with moderate-intense expression in $20-50 \%$ of the samples. Similar distribution was seen among T-cell lymphomas. CD205 exhibited both membranous and cytoplasmic localization in the lymphoma cells; expression varied within individual cases (Online Supplementary Figure S1).

\section{MEN1309/0BT076 has in vitro anti-tumor activity in diffuse large B-cell lymphoma}

Based on the expression data, we exposed 42 B-cell lymphoma cell lines derived from DLBCL, MCL, MZL, and CLL, to the anti-CD205 ADC MEN1309/OBT076 (Online Supplementary Table S2). The compound demonstrated a strong anti-tumor activity with a median $\mathrm{IC}_{50}$ of $200 \mathrm{pM}$ (95\% CI: $15 \mathrm{pM}-20 \mathrm{nM}$ ). The control ADC, human IgG conjugated to the DM4 toxin, was 100 times less active (20 nM; 95\%CI: 13-70 nM) (Table 2). The anti-tumor activity of MEN1309/OBT076 was mostly cytotoxic: apoptosis induction was observed in 25 of $42(59.5 \%)$ cell lines at a concentration of $1 \mathrm{nM}$. Cell cycle analysis further 
A

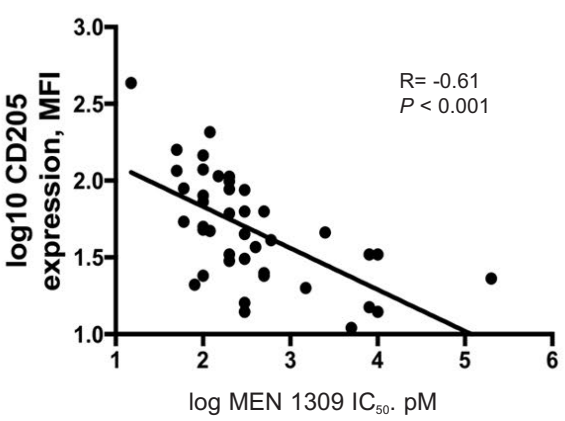

C

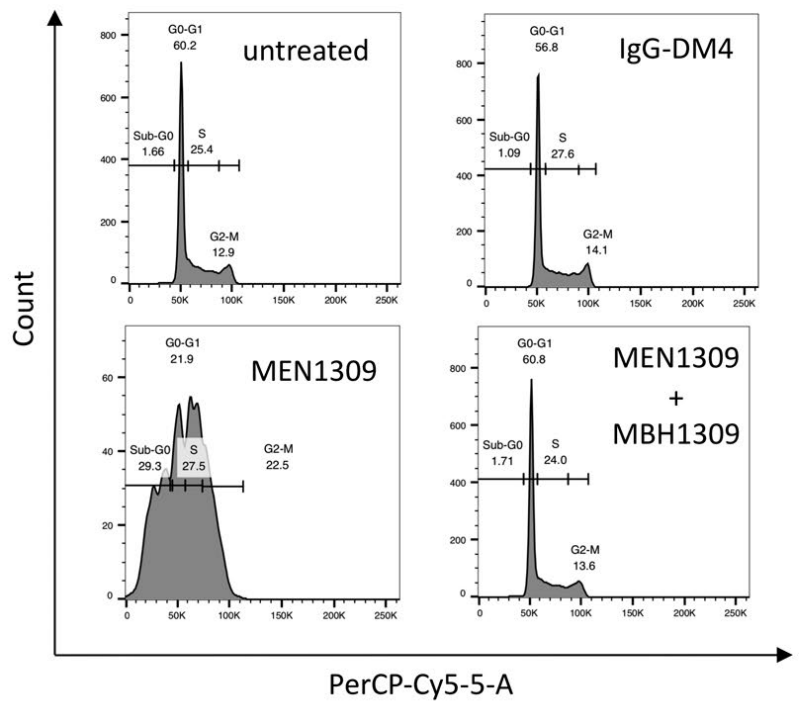

B

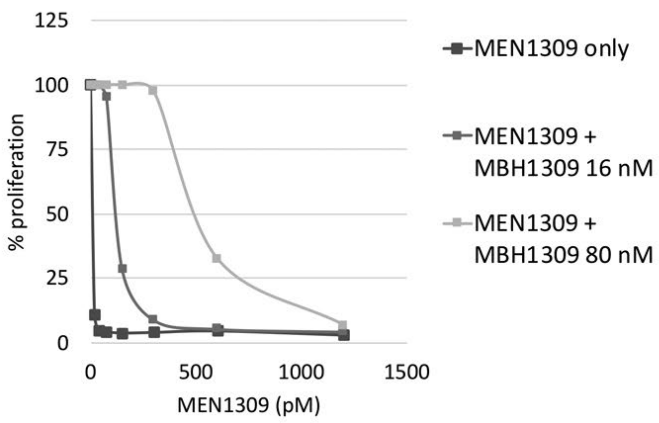

D

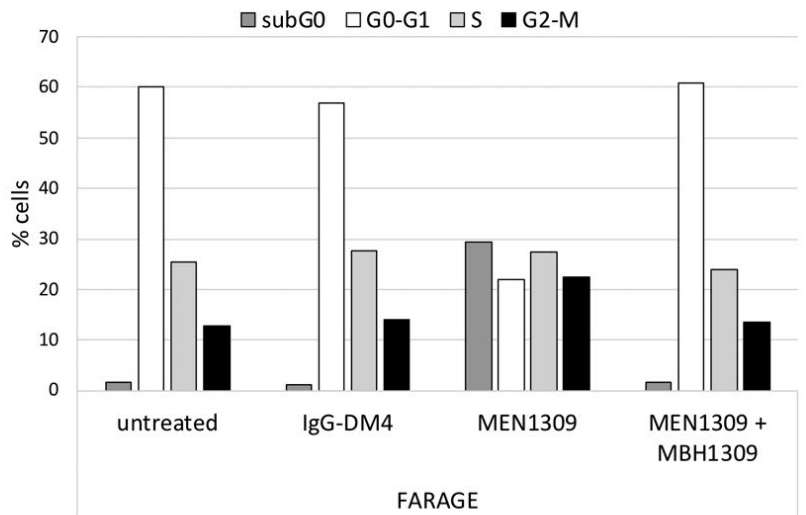

Figure 1. The antitumor of MEN1309/OBT076 is correlated to the expression of its target. (A) Correlation in all B-cell lymphoma. $y$-axis: Log10 MFI values of CD205 as measured by flow cytometry. x-axis: Log10 IC I $_{50}$ values of MEN1309/OBT076 (pM). (B) FARAGE cell lines were treated with MEN1309/OBTO76 (0-1.2pM and two different concentrations of MBH1309: 16 and 80nM). (C) Representative histogram of cell cycle distribution in FARAGE cells treated with 100pM of IgG-DM4, MEN1309/OBT076 alone and in combination with MBH1309 (72 hours). (D) Cell cycle distribution after exposure with IgG-DM4, MEN1309/OBT076 or combination with $\mathrm{MBH} 1309$. Graph plots show mean \pm standard deviation for values from two biological replicates. MFI: mean fluorescence intensity.

confirmed the cytotoxic activity of MEN1309/OBT076. Four cell lines (Farage, OCI-LY-1, SU-DHL-4 and SU-DHL10) were treated with MEN1309/OBT076 (low concentration and high concentration, reflecting $\mathrm{IC}_{50}$ values for each cell line), and with IgG-DM4 and the unconjugated antiCD205 antibody for 72 hours. In all the cases, MEN1309/OBT076 induced a strong sub-G0 arrest in the cell lines, while the effect of the two control antibodies (IgG-DM4 and naked antibody MBH-1309) was minimal (Online Supplementary Figure S2). No differences in antitumor activity were seen according to DLBCL cell of origin, lymphoma histotype, the presence of MYC translocation or BCL2 translocation or TP53 inactivation (Online Supplementary Table S2). The ADC MEN1309/OBT076 did not exert any apoptotic effects against PBMC from two healthy donors who had CD205 expression on their CD19+ $\mathrm{B}$ cells similar to one the most sensitive DLBCL cell lines (171 and 111 vs. 106 of the OCI-LY-10) (Online Supplementary Figure S3).

\section{MEN1309/0BT076 anti-tumor activity was highly correlated with CD205 expression in diffuse large B-cell lymphoma cell lines}

We then evaluated whether the activity of
MEN1309/OBT076 was affected by the expression levels of its target. Sensitivity to the ADC was correlated with the cell surface CD205 expression measured by flow cytometry in 41 lymphoma cell lines (Online Supplementary Table S3), as shown by the negative correlation between $\mathrm{IC}_{50}$ values and the mean fluorescence intensity values $(P<0.0001)$ (Figure 1A).

Focusing on 23 DLBCL cells, we also assessed the correlation between sensitivity and RNA expression of the LY75 gene, coding for CD205, as measured by gene expression profiling or real-time polymerase chain reaction (RT-PCR) (Online Supplementary Table S3). The expression levels obtained with the two modalities were positively correlated between them $(P<0.001)$ and with cell membrane protein expression $(P<0.001)$ (Online Supplementary Figure S4). Similar to protein expression, also RNA levels were negatively correlated with $\mathrm{IC}_{50}$ values $(P<0.001)$ (Online Supplementary Figure S5).

The LY75 gene, coding for CD205, is known to give rise to intergenically spliced transcripts with its centromeric neighboring gene CD302..$^{5}$ MEN1309/OBT076 activity significantly correlated with the expression of two CD205-CD302 intergenically spliced transcripts, measured by RT-PCR, indicating that this phenomenon does 
Table 1. Prevalence of CD205 expression in hematologic cancers as demonstrated by immunohistochemistry (IHC) on tumor microarrays.

\begin{tabular}{|c|c|c|c|}
\hline & N. of samples & & \\
\hline & & $\geq 1+(\%)$ & $2-3+(\%)$ \\
\hline Lymphoma & & & \\
\hline B-cell neoplasms & & & \\
\hline B cell lymphoma (undefined) & 302 & 71 & 37 \\
\hline Diffuse large B-cell lymphoma & 82 & 73 & 28 \\
\hline Burkitt's lymphoma & 7 & 29 & 0 \\
\hline Follicular lymphoma & 16 & 81 & 23 \\
\hline Lymphoplasmacytic lymphoma & 13 & 31 & 23 \\
\hline Mantle cell lymphoma & 7 & 71 & 29 \\
\hline Mucosa-associated lymphatic tissue lymphoma & 40 & 88 & 50 \\
\hline Small lymphocytic lymphoma & 3 & 0 & 0 \\
\hline T-cell rich B-cell lymphoma & 3 & 33 & 33 \\
\hline Hodgkin lymphoma & & & \\
\hline Hodgkin lymphoma (undefined) & 5 & 80 & 20 \\
\hline Lymphocyte depleted Hodgkin lymphoma & 2 & 50 & 50 \\
\hline Lymphocyte predominant Hodgkin lymphoma & 7 & 57 & 14 \\
\hline Mixed cellularity & 15 & 100 & 7 \\
\hline Nodular sclerosis & 5 & 100 & 60 \\
\hline T-cell and NK-cell neoplasms & & & \\
\hline T-cell lymphoma (undefined) & 68 & 59 & 28 \\
\hline Anaplastic large cell lymphoma & 8 & 88 & 50 \\
\hline Angioimmunoblastic T-cell lymphoma & 7 & 86 & 43 \\
\hline Peripheral T-cell lymphoma & 3 & 100 & 0 \\
\hline $\begin{array}{l}\text { Leukemia } \\
\text { Acute myeloid leukemia }\end{array}$ & 26 & 62 & 58 \\
\hline Myeloma & & & \\
\hline Multiple myeloma & 14 & 100 & 79 \\
\hline
\end{tabular}

$\mathrm{N}$ : number; NK: natural killer

not reduce the anti-tumor activity of the compound $(\mathrm{R}=-$ 0.78 and $\mathrm{R}=-0.72, P<0.001$ ) (Online Supplementary Table $S 3)$, as well $\mathrm{CD} 302$ itself ( $\mathrm{R}=0.67, P=0.001$ ).

Finally, to further assess the specificity of MEN1309/OBT076, we treated cells with the ADC with or without the addition of MBH-1309, the naked antiCD205 antibody. The latter molecule had no anti-tumor activity by itself (Online Supplementary Figure S6), but it decreased the activity of MEN1309/OBT076 in a dose dependent manner, as shown both in terms of $\mathrm{IC}_{50}$ and of cell cycle analysis (Figure 1B-D).

\section{MEN1309/0BT076 demonstrates in vivo anti-tumor activity in DLBCL}

To confirm the observed in vitro MEN1309/OBT076 anti-lymphoma activity, we performed an in vivo xenograft experiment with the OCI-LY-10 model of DLBCL. Cohorts of mice were treated with vehicle control, IgGDM4 $(5 \mathrm{mg} / \mathrm{kg}$, once every 3 weeks), or MEN1309/OBT076 $(1.25,2.5$ or $5 \mathrm{mg} / \mathrm{kg}$ once every 3 weeks) (Figure 2A). Low activity was observed with MEN1309/OBT076 $1.25 \mathrm{mg} / \mathrm{kg}$ (day [D] 7, $P=0.012$ ) and with IgG-DM4 (D21, $P=0.049 ; \quad \mathrm{D} 28, \quad P=0.046$ ). MEN1309/OBT076 at $2.5 \mathrm{mg} / \mathrm{kg}$ delayed tumor growth versus control $(\mathrm{D} 21, P=0.039)$. MEN1309/OBT076 at 5 $\mathrm{mg} / \mathrm{kg}$ eradicated tumors in all mice with a single dose, highlighted by highly significant differences in tumor vol- ume versus control mice (D7, D21, D28; $P<0.01)$. Tumor eradication led to an increase in the survival of the mice. While all other groups reached endpoint by D35, the MEN1309/OBT076 $5 \mathrm{mg} / \mathrm{kg}$ group skipped the second planned treatment at D21 because there was no sign of tumors, and mice remained cured up to two months later (Kaplan-Meier, survival analysis, $P<0.0001$ ) (Figure $2 B$ ). One mouse belonging to the $5 \mathrm{mg} / \mathrm{kg}$ group showed tumor re-growth (D28 and D35), and a second MEN1309/OBT076 injection (D43) conferred tumor remission for the second time (D50 and D57).

\section{MEN1309/0BT076 is synergistic with other targeted agents}

MEN1309/OBT076 was then combined with targeted agents in four ABC-DLBCL cell lines (TMD8, HBL1, OCILY-10 and U2932) (Table 3). The most effective combinations were with the anti-CD20 monoclonal antibody rituximab (synergistic in all four), and with the BCL2 inhibitor venetoclax (synergistic in three, additive in one). Both combinations determined increased cell counts as shown by increased percentage of cells in sub-G0 phase during a cell cycle experiment (Online Supplementary Figure S7). Immunoblotting analyses of two ABC-DLBCL cell lines (TMD8 and OCI-LY-10) after exposure to MEN1309/OBT076 provided hints on the mechanism underlining the synergism (Figure 3). Protein levels of anti- 
apoptotic proteins were affected by exposure to MEN1309/OBT076, which induced a downregulation of MCL1 protein and upregulation of BCL2 and BCX-L. The $\mathrm{ADC}$ also determined an upregulation of CD20 protein levels. All the changes were maintained when MEN1309/OBT076 was given in combination with the BCL2 inhibitor or the anti-CD20 monoclonal antibody, and not seen after these two drugs were given as single agents. PARP1 cleavage confirmed apoptosis induction.

The combinations with the PI3K- $\delta$ inhibitor idelalisib was additive in two cell lines and of no benefit in the other two, while the immunomodulatory agent lenalidomide was additive in one cell line and of no benefit in the remaining three.
A

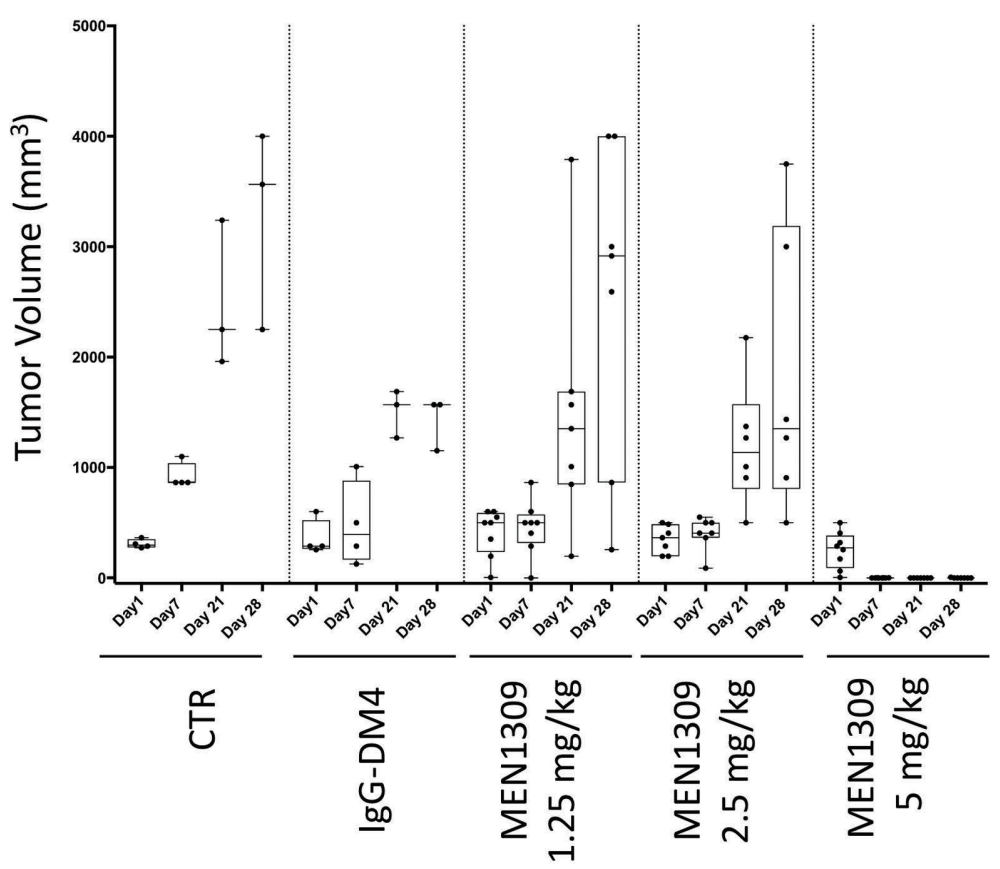

B

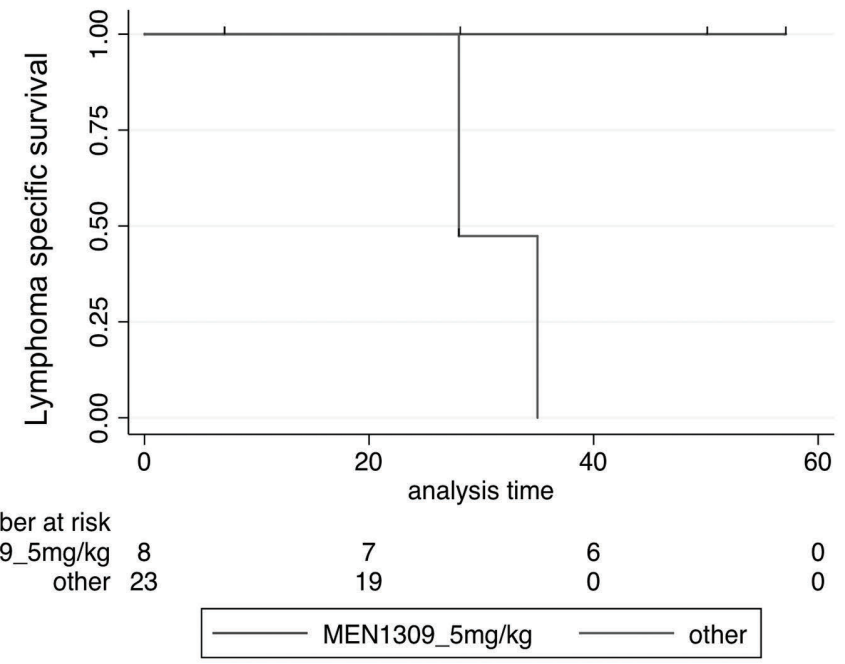

Figure 2. MEN1309/OBT076 as single agent has in vivo antitumor activity in the activated B-cell like diffuse large B-cell lymphoma $\mathrm{OCl}$ LY10 xenograft model. Treatment with MEN1309/OBTO76 and IgG-DM4 started when tumors became visible $\left(100 \mathrm{~mm}^{3}\right)$. (A) Control (CTR) group, $\mathrm{n}=4$; IgG-DM4 group, $\mathrm{n}=4$; MEN1309/OBTO76 $1.25 \mathrm{mg} / \mathrm{kg}, \mathrm{n}=8$ MEN1309/OBTO76 $2.5 \mathrm{mg} / \mathrm{kg}, \mathrm{n}=7$ MEN1309/OBT076 $5 \mathrm{mg} / \mathrm{kg}, \mathrm{n}=8$. In each box-plot, the line in the middle of the box represents the median and the box extends from the $25^{\text {th }}$ to the $75^{\text {th }}$ percentile (IQ: interquartile range). Each dot represent a mouse. $x$ axis: days of treatment. $y$-axis: tumor volume expressed in $\mathrm{mm}^{3}$. (B) Kaplan-Meier LSS (lymphoma specific survival) MEN1309/OBT076 (5 mg/kg) versus all other groups together. $P<0.0001$.

Table 2. Anti-tumor activity of MEN1309/0BT076 and IgG-DM4 in B-cell lymphoma cell lines.

\begin{tabular}{|c|c|c|c|c|c|}
\hline & \multirow[b]{2}{*}{ N. of cell lines } & \multicolumn{2}{|c|}{ MEN1309/0BT076 } & \multicolumn{2}{|c|}{ IgG-DM4 } \\
\hline & & Median $\mid \mathbf{C}_{50}$ & $95 \% \mathrm{CI}$ & Median IC F0 & $95 \%$ CI \\
\hline GCB-DLBCL & 15 & $200 \mathrm{pM}$ & $118 \mathrm{pM}-4163 \mathrm{pM}$ & $30 \mathrm{nM}$ & $20-38 \mathrm{nM}$ \\
\hline ABC-DLBCL & 8 & $300 \mathrm{pM}$ & 200 pM-3612 pM & $30 \mathrm{nM}$ & $20-40 \mathrm{nM}$ \\
\hline MCL & 10 & $100 \mathrm{pM}$ & $86-1883 \mathrm{pM}$ & $15 \mathrm{nM}$ & $15-54 \mathrm{nM}$ \\
\hline MZL & 6 & $110 \mathrm{pM}$ & $55-465 \mathrm{pM}$ & $17.5 \mathrm{nM}$ & $15-38.5 \mathrm{nM}$ \\
\hline CLL & 2 & $310 \mathrm{pM}$ & n.d. & $34 \mathrm{nM}$ & n.d. \\
\hline
\end{tabular}

GCB-DLBCL: germinal center B-cell type diffuse large B-cell lymphoma; ABC-DLBCL: activated B-cell like diffuse large B-cell lymphoma; MCL: mantle cell lymphoma; MZL: marginal zone lymphoma; CLL: chronic lymphocytic leukemia; n.d.: not determined; N: number; CI: confidence interval. 
Combined MEN1309/0BT076 and rituximab showed higher in vivo anti-tumor activity than the single agents

The in vitro synergism with rituximab was validated in vivo using an ABC-DLBCL lymphoma model, OCI-LY-10 (Figure 4). Mice were divided in four groups of ten animals each and were treated with MEN1309/OBT076 (2.5 $\mathrm{mg} / \mathrm{kg}$ IV, D1 and D12), or rituximab (3 mg/kg IV on D1; $5 \mathrm{mg} / \mathrm{kg}$ IV on D12), or MEN1309/OBT076 plus rituximab (same schedule as single agents), or with vehicle only (IV).

Single agents and combinations were more active $(P<0.05)$ than controls (MEN1309/OBT076, rituximab, MEN1309/OBT076 plus rituximab, starting at D10). The combination of MEN1309/OBT076 with rituximab gave significant differences at D17 $(P<0.05)$ versus both single agent arms and tumor eradication. At the used doses, MEN1309/OBT076 presented higher anti-lymphoma activity compared to rituximab, although this was not statistically significant $(P=0.06, \mathrm{D} 17)$.

\section{Discussion}

Here we have shown CD205 expression in hematologic cancers, including lymphomas. Results show that targeting this antigen with a first-in-class anti-CD205 ADC, MEN1309/OBT076, had both in vitro and in vivo anti-tumor activity in lymphomas. The novel ADC had an anti-tumor activity that was highly correlated with expression of its target CD205 and reached synergism when combined with other targeted agents.
Immunohistochemistry analysis of histological sections of lymphomas, myeloma and leukemia clinical specimens showed that a large percentage of cases $(>70 \%)$ express CD205. This is in agreement with recently reported data in series of 100 DLBCL and 33 follicular lymphomas in which CD205 positivity was detected in $60 \%$ and $79 \%$ of the cases, respectively. ${ }^{8} \mathrm{CD} 205$ has also been detected at high expression level in solid tumors (gastric, pancreatic, bladder, breast and colon).

CD205 is a type I transmembrane glycoprotein and a Ctype lectin receptor, which undergoes endocytosis ${ }^{7}$ and thus, can be exploited as a novel target for ADC. The fully humanized ADC MEN1309/OBT076 binds to CD205 with high affinity and is rapidly internalized in the cells, delivering the microtubule disruptor DM4 as payload.' Based on expression pattern in lymphoma clinical specimens, we assessed the anti-tumor activity of MEN1309/OBT076 in lymphoma cell lines. The ADC presented a very strong anti-tumor activity with subnanomolar $\mathrm{IC}_{50}$ values. Of clinical relevance, at least in cell lines, sensitivity to MEN1309/OBT076 was not affected by DLBCL cell of origin, MYC/BCL2 or TP53 status. The in vitro results were confirmed in a xenograft model using an ABC DLBCL cell line. A single dose of MEN1309/OBT076 at $5 \mathrm{mg} / \mathrm{kg}$ led to a complete remission that lasted for at least two months. A second dose was able to obtain a second remission in the only tumor that presented a regrowth. These data are similar to recent observations in solid tumor models exposed to MEN1309/OBT076. The strong in vitro and in vivo data, and the notion that its target
OCI-LY-10

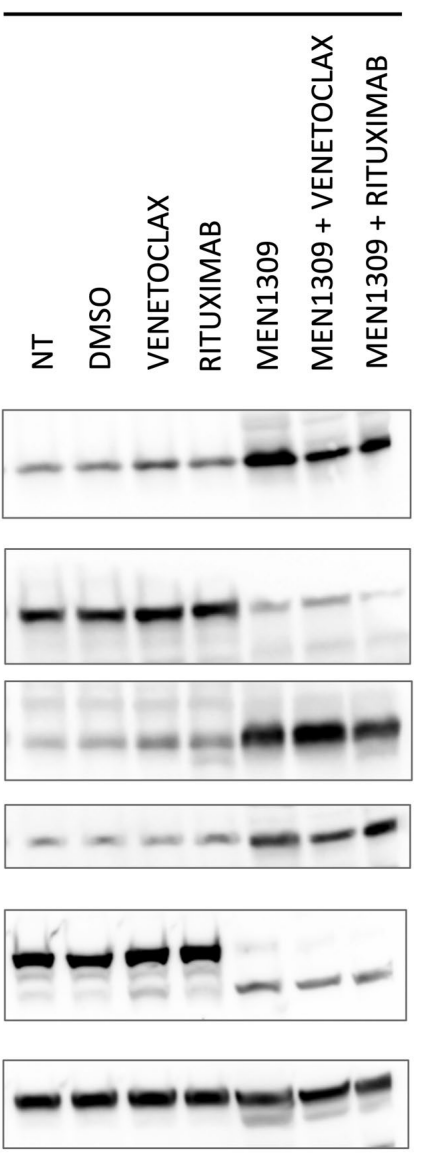

TMD8
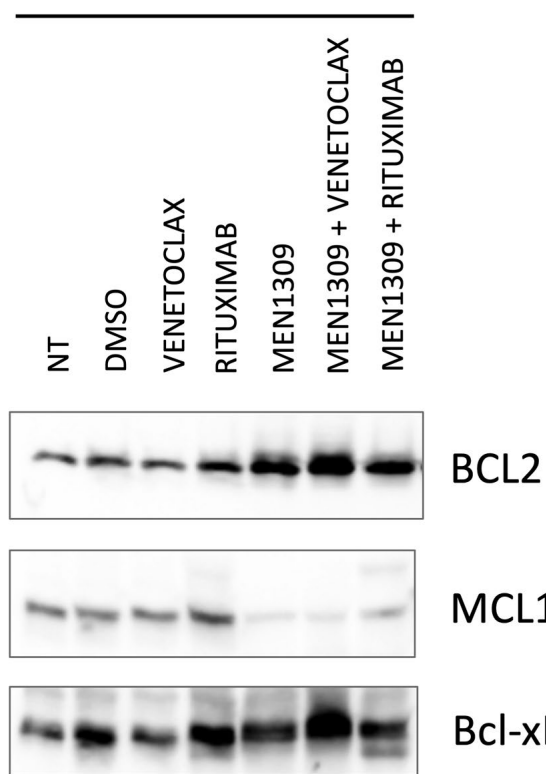

$B c l-x L$

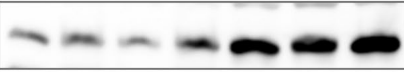

CD20

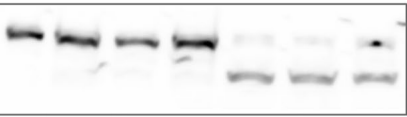

PARP1

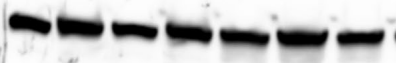

\section{beta-tubulin}

Figure 3. MEN1309/ОВT076 combined with the BCL2 inhibitor venetoclax and the anti-CD20 monoclonal antibody rituximab exerts cytotoxicity downregulating MCL1, cleaving PARP and upregulating CD20 protein. Two cell lines were exposed to MEN1309/OBT076 (1nM), venetoclax (100 nM), rituximab (20 $\mu \mathrm{g} / \mathrm{mL}$ ) or the combination of the agents for 72 hours. $\beta$-tubulin was used as a loading control. Figure is representative of two independent experiments. 


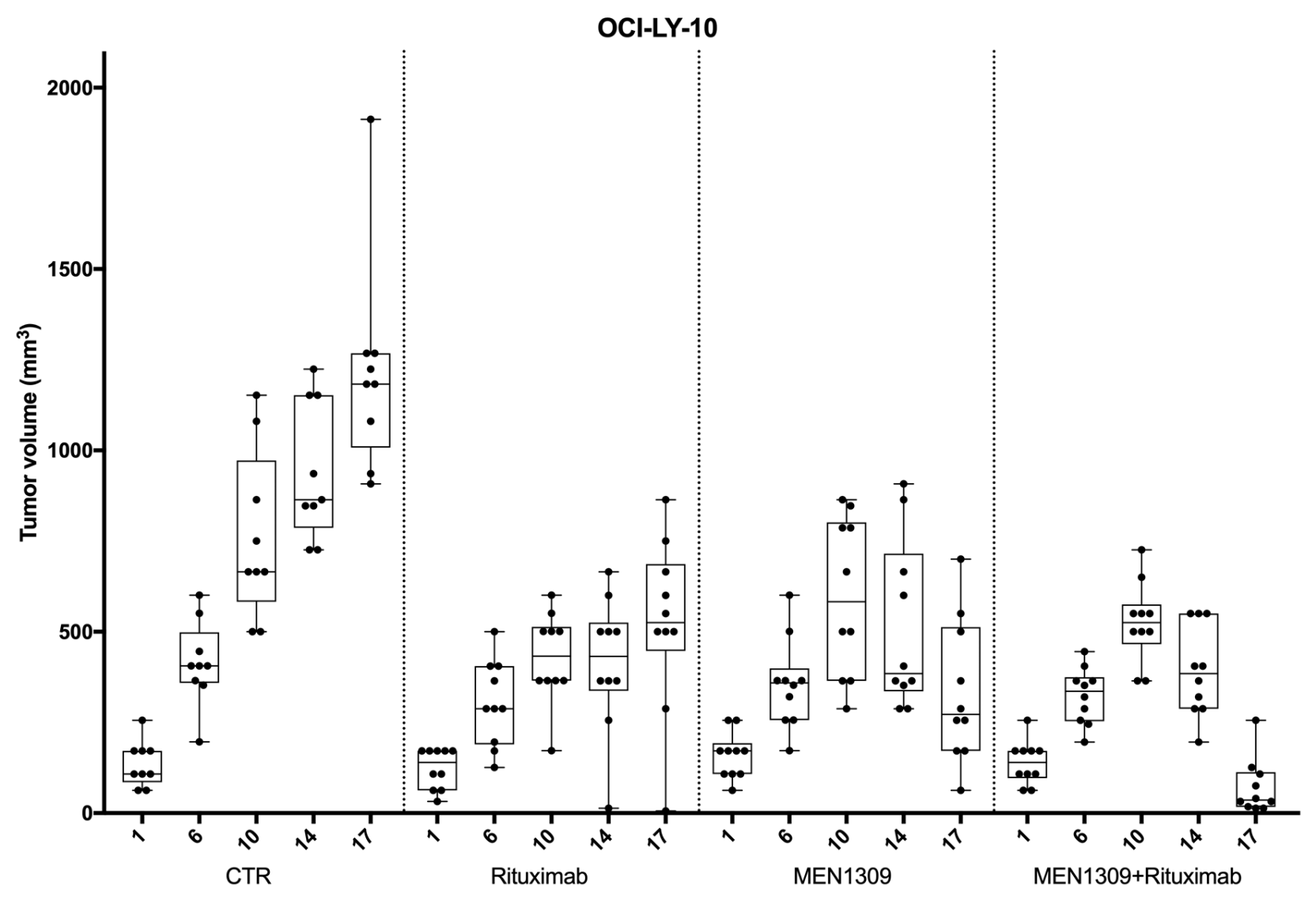

Figure 4. Combined MEN1309/OBTO76 and rituximab shown higher in vivo anti-tumor activity than the single agents in the activated B-cell like diffuse large B-cell lymphoma OCI-LY10 xenograft model. Treatment with MEN1309/OBTO76 ( $2.5 \mathrm{mg} / \mathrm{kg})$, rituximab (5 mg/kg), their combination or vehicle started when tumors became visible $\left(>100 \mathrm{~mm}^{3}\right)$. In each box-plot, the line in the middle of the box represents the median and the box extends from the $25^{\text {th }}$ to the $75^{\text {th }}$ percentile. CTR: control; IQ: interquartile range. $y$-axis: tumor volume in $\mathrm{mm}^{3}$; $\mathrm{x}$-axis: days of treatment.

is not shared by other antibody or cell ${ }^{1,14-16}$ based therapies, make MEN1309/OBT076 a very interesting novel compound for lymphoma patients.

We did not observe toxicity in our in vivo models, in agreement with reports from in vivo studies of solid tumor models,' but MBH1309/OBT076 does not significantly cross-react to the mouse CD205. ${ }^{7}$ Toxicity studies performed on cynomolgus monkeys, whose CD205 crossreacts to $\mathrm{MBH} 1309 / \mathrm{OBT} 076$, have shown moderate toxicity with neutropenia as main adverse event. ${ }^{7}$ There is no difference in CD205 expression pattern between humans and cynomolgus monkeys across 33 different normal tissues, with a membranous/cytoplasmic staining of mononuclear cells in several tissues, including lymph nodes and spleen. ${ }^{7}$ Here, we exposed human PBMC from two healthy donors to the MEN1309; no cytotoxicity was observed despite the fact that the B cells expressed CD205 levels similar to those observed in a sensitive DLBCL cell line. One reason could be that circulating B cells are not proliferating and are thus less sensitive to the payload. This would agree with the suggested explanation of the low toxicity observed in CD205 positive normal tissues in cynomolgus monkeys.?

The anti-tumor activity of MEN1309/OBT076 appeared to be mediated by the ADC binding to its target as shown by the very high correlation between $\mathrm{IC}_{50}$ values and target expression, and by competition experiments with a
Table 3. In vitro assessment of MEN1309/OBT076 combinations with targeted agents.

\begin{tabular}{lccc} 
Cell line & Drug 2 & $\begin{array}{c}\text { Median } \\
\text { combination index }\end{array}$ & $\begin{array}{c}95 \% \\
\text { CI }\end{array}$ \\
HBL1 & Idelalisib & $0.96^{\wedge}$ & $0.78-1$ \\
OCI-LY-10 & Idelalisib & 2.51 & $1.91-2.76$ \\
\hline TMD8 & Idelalisib & 1.14 & $1.02-1.32$ \\
U2932 & Idelalisib & $0.91^{\wedge}$ & $0.7-1.4$ \\
\hline HBL1 & Lenalidomide & 1.31 & $0.92-2.34$ \\
OCI-LY-10 & Lenalidomide & 1.46 & $1.19-1.58$ \\
\hline TMD8 & Lenalidomide & $0.94^{\wedge}$ & $0.64-1.44$ \\
U2932 & Lenalidomide & 1.47 & $0.97-2.08$ \\
\hline HBL1 & Rituximab & $0.57^{*}$ & $0.51-0.67$ \\
OCI-LY-10 & Rituximab & $0 . *^{*}$ & $0.42-2.37$ \\
\hline TMD8 & Rituximab & $0.27^{*}$ & $0.22-0.33$ \\
U2932 & Rituximab & $0.47^{*}$ & $0.39-0.8$ \\
\hline HBL1 & Venetoclax & $0.51^{*}$ & $0.32-0.7$ \\
OCI-LY-10 & Venetoclax & $0.86^{*}$ & $0.68-0.53$ \\
\hline TMD8 & Venetoclax & $1.04^{\wedge}$ & $0.75-1.52$ \\
U2932 & Venetoclax & $0.49^{*}$ & $0.32-0.83$ \\
\hline
\end{tabular}

Cell lines were exposed to increasing concentrations of MEN1309/OBT076 and/or of the other compound (drug 2) for 72 hours. Based on the Chou-Talalay Combination Index, the effect of the combinations was defined as beneficial if synergistic $(<0.9)$ or additive (0-9-1.1). Cl: confidence interval. 
naked antibody recognizing the same epitope targeted by MEN1309/OBT076. No direct anti-tumor effect is reported for the naked antibody MEN1309/OBT076. It is noteworthy that the expression level of CD205 in cell lines measured at the level of mRNA, total protein or cell surface protein correlated with the sensitivity to MEN1309/OBT076. Furthermore, a clinical DLBCL specimen-derived CD205 signature also correlated with the in vitro response to the drug. In contrast, the expression of two annotated CD205-CD302 intergenically spliced transcripts $^{5}$ did not affect the anti-tumor activity of MEN1309/OBT076.

Finally, MEN1309/OBT076 was beneficially combined with other targeted agents, especially the BCL2 inhibitor venetoclax and the anti-CD20 monoclonal antibody rituximab. The latter combination, that had shown the best in vitro results, was successfully validated in vivo using a xenograft model. ${ }^{17}$ Interesting clinical data are also already available for combinations with other ADC. ${ }^{18,19}$ The mechanisms leading to better anti-tumor activity can differ. The concomitant exposure of lymphoma cells to rituximab and to the anti-CD37 ADC IMGN529/Debio1562 leads to improved anti-lymphoma activity due to an increased internalization of the latter. ${ }^{20}$ The benefit of combining the anti-CD22 inotuzumab ozogamicin (CMC-544) with rituximab could be due to both an early upregulation of CD20 with increased direct cytotoxicity of the antiCD2021 or to the addition of the direct (inotuzumab ozogamicin), complement and/or cellular dependent (rituximab) mechanisms of actions of the compounds. ${ }^{22}$ Here we observed that exposure to MEN1309/OBT076 was followed by upregulation of the rituximab target CD20 and downregulation of the anti-apoptotic protein MCL1, known to counteract the activity of venetoclax in DLBCL cells, ${ }^{23}$ two changes that could support the observed synergisms. Moreover, the addition of venetoclax also counteracts the BCL2 upregulation seen after the ADC.

In conclusion, the first-in-class ADC targeting CD205, MEN1309/OBT076, demonstrated strong pre-clinical antitumor activity in lymphoma. Our data sustain the ongoing clinical CD205-Shuttle study (clinicaltrials.gov identifier: NCT03403725) of MEN1309/OBT076 as single agent, and provide the rationale for further investigations also in combination therapy.

\section{References}

1. Moek KL, de Groot DJA de Vries EGE, Fehrmann RSN. The antibody-drug conjugate target landscape across a broad range of tumour types. Ann Oncol. 2017; 28(12):3083-3091

2. Shrimpton RE, Butler M, Morel AS, Eren E, Hue SS, Ritter MA. CD205 (DEC-205): a recognition receptor for apoptotic and necrotic self. Mol Immunol. 2009, 46(6):1229-1239.

3. Cao L, Shi X, Chang H, Zhang Q, He Y. pHDependent recognition of apoptotic and necrotic cells by the human dendritic cell receptor DEC205. Proc Natl Acad Sci U S A. 2015;112(23):7237-7242.

4. Butler M, Morel AS, Jordan WJ, et al. Altered expression and endocytic function of CD205 in human dendritic cells, and detection of a CD205-DCL-1 fusion protein upon dendritic cell maturation. Immunology. 2007;120(3):362-371

5. Kato M Khan S, Gonzalez N, et al. Hodgkin's lymphoma cell lines express a fusion protein encoded by intergenically spliced mRNA for the multilectin receptor DEC-205 (CD205) and a novel C-type lectin receptor DCL-1. J Biol Chem. 2003, 278(36):34035-34041.

6. Uhlen M, Fagerberg L, Hallstrom BM, et al. Proteomics. Tissue-based map of the human proteome. Science. 2015; 347(6220):1260419.

7. Merlino G, Fiascarelli A, Bigioni $M$, et al. MEN1309/OBT076, a first-in-class antibody-drug conjugate targeting CD205 in solid tumors. Mol Cancer Ther. 2019;18(9):1533-1543

8. Canzonieri V, Gattei V, Spina M, et al CD205, a target antigen for a novel antibody drug conjugate (ADC): evaluation of antigen expression on non-Hodgkin lymphoma (NHL). I Clin Oncol. 2017;35(15 Suppl):e14039-e14039.

9. Garralda E, Tabernero J, Garcia VM, De Miguel MJ, Plummer ER, Jerusalem GHM. CD205-Shuttle study: a first-in-human trial of MEN1309/OBT076 an ADC targeting CD205 in solid tumor and NHL. J Clin Oncol. 2018;36(15):TPS2606.

10. Tarantelli C, Gaudio E, Arribas AJ, et al. POR309 is a novel dual PI3K/mTOR inhibitor with preclinical antitumor activity in lymphomas as a single agent and in combination therapy. Clin Cancer Res. 2018;24(1):120-129.

11. Hicks SW, Tarantelli C, Wilhem A, et al. The novel CD19-targeting antibody-drug conjugate huB4-DGN462 shows improved antitumor activity compared to SAR3419 in CD19-positive lymphoma and leukemia models. Haematologica. 2019;104(8):16331639.

12. Boi M, Gaudio E, Bonetti P, et al. The BET bromodomain inhibitor OTX015 affects pathogenetic pathways in preclinical B-cell tumor models and synergizes with targeted drugs. Clin Cancer Res. 2015;21(7):16281638.

13. Chou TC. Preclinical versus clinical drug combination studies. Leuk Lymphoma. 2008;49(11):2059-2080

14. Younes A, Ansell S, Fowler N, et al. The landscape of new drugs in lymphoma. Nat Rev Clin Oncol. 2017;14(6):335-346.

15. Pianko MJ, Moskowitz AJ, Lesokhin AM. Immunotherapy of lymphoma and myeloma: facts and hopes. Clin Cancer Res. 2018; 24(5):1002-1010.

16. Chow VA, Shadman M, Gopal AK. Translating anti-CD19 CAR T-cell therapy into clinical practice for relapsed/refractory diffuse large B-cell lymphoma. Blood. 2018; 132(8):777-781.
17. Karmali R, Kimby E, Ghielmini M, Flinn IW, Gordon LI, Zucca E. Rituximab: a benchmark in the development of chemotherapyfree treatment strategies for follicular lymphomas. Ann Oncol. 2018;29(2):332-340.

18. Advani RH, Lebovic D, Chen A, et al. Phase I study of the anti-CD22 antibody-drug conjugate pinatuzumab vedotin with/without rituximab in patients with relapsed/refractory B-cell non-Hodgkin lymphoma. Clin Cancer Res. 2017;23(5):1167-1176.

19. Fayad L, Offner F, Smith MR, et al. Safety and clinical activity of a combination therapy comprising two antibody-based targeting agents for the treatment of non-Hodgkin lymphoma: results of a phase I/II study evaluating the immunoconjugate inotuzumab ozogamicin with rituximab. J Clin Oncol. 2013;31(5):573-583

20. Hicks SW, Lai KC, Gavrilescu LC, et al. The antitumor activity of IMGN529, a CD37-targeting antibody-drug conjugate, is potentiated by rituximab in non-Hodgkin lymphoma models. Neoplasia. 2017;19(9):661-671.

21. Takeshita A, Yamakage N, Shinjo K, et al. CMC-544 (inotuzumab ozogamicin), an anti-CD22 immuno-conjugate of calicheamicin, alters the levels of target molecules of malignant B-cells. Leukemia. 2009; 23(7):1329-1336.

22. Dijoseph JF, Dougher MM, Kalyandrug LB, et al. Antitumor efficacy of a combination of CMC-544 (inotuzumab ozogamicin), a CD22-targeted cytotoxic immunoconjugate of calicheamicin, and rituximab against nonHodgkin's B-cell lymphoma. Clin Cancer Res. 2006;12(1):242-249.

23. Klanova M, Andera L, Brazina I, et al. Targeting of BCL2 family proteins with ABT-199 and homoharringtonine reveals BCL2- and MCL1-dependent subgroups of diffuse large B-cell lymphoma. Clin Cancer Res. 2016;22(5):1138-1149. 